\title{
Single Nucleotide Polymorphisms in B-Genome Specific UDP-Glucosyl Transferases Associated with Fusarium Head Blight Resistance and Reduced Deoxynivalenol Accumulation in Wheat Grain
}

\author{
Pallavi Sharma, Manu P. Gangola, Chen Huang, H. Randy Kutcher, Seedhabadee Ganeshan, and Ravindra N. Chibbar ${ }^{\dagger}$
}

All authors: Department of Plant Sciences, University of Saskatchewan, 51 Campus Drive, Saskatoon, S7N 5A8, Saskatchewan, Canada. Current address of P. Sharma: Central University of Jharkhand, Ranchi, Jharkhand, India.

Current address of S. Ganeshan: Biotechnology Laboratories, Saskatchewan Research Council, Saskatoon, Saskatchewan, Canada. Accepted for publication 27 August 2017.

\begin{abstract}
An in vitro spike culture method was optimized to evaluate Fusarium head blight (FHB) resistance in wheat (Triticum aestivum) and used to screen a population of ethyl methane sulfonate treated spike culturederived variants (SCDV). Of the $134 \mathrm{SCDV}$ evaluated, the disease severity score of 47 of the variants was $\leq 30 \%$. Single nucleotide polymorphisms (SNP) in the UDP-glucosyltransferase (UGT) genes, TaUGT-2B, TaUGT-3B, and TaUGT-EST, differed between AC Nanda (an FHB-susceptible wheat variety) and Sumai-3 (an FHB-resistant wheat cultivar). SNP at 450 and $1,558 \mathrm{bp}$ from the translation initiation site in TaUGT-2B and TaUGT-3B,

respectively were negatively correlated with FHB severity in the SCDV population, whereas the SNP in TaUGT-EST was not associated with FHB severity. Fusarium graminearum strain M7-07-1 induced early expression of TaUGT-2B and TaUGT-3B in FHB-resistant SCDV lines, which were associated with deoxynivalenol accumulation and reduced FHB disease progression. At 8 days after inoculation, deoxynivalenol concentration varied from $767 \mathrm{ppm}$ in FHB-resistant variants to 2,576 ppm in FHB-susceptible variants. The FHB-resistant SCDV identified can be used as new sources of FHB resistance in wheat improvement programs.
\end{abstract}

Fusarium head blight (FHB) or scab is a devastating disease of wheat and other small grain cereals that has worldwide economic and health impacts (Rawat et al. 2016). The disease is mainly caused by Fusarium graminearum Schwabe [teleomorph: Gibberella zeae (Schw.) Petch)] (Arruda et al. 2016). FHB not only affects grain development and yield, but also contaminates infected grain with trichothecene mycotoxins, especially deoxynivalenol (DON) and nivalenol (NIV), which limits use of the grain for food and feed (Bai and Shaner 1994; Cho et al. 2012; Ichinoe et al. 1983). In livestock, DON is associated with feed refusal (Charmley and Trenholm 2015), whereas in humans, it may cause gastrointestinal symptoms including vomiting and depression of the immune system (Maresca et al. 2002; Pestka et al. 2004). Trichothecenes such as DON are acutely phytotoxic and act as virulence factors on susceptible host plants. In Canada, progression of FHB is of great concern for wheat production and grain quality. In 2015, FHB affected 34\% of the spring wheat crops surveyed in Saskatchewan and $77 \%$ in Manitoba, the major spring wheat producing provinces of Canada (Brar et al. 2016; Henriquez et al. 2016).

FHB resistance has been categorized into five classes: Type I (resistance to initial infection), Type II (resistance to disease progression within the spike), Type III (resistance to DON production), Type IV (resistance to kernel infection) and Type V (tolerance) (Mesterházy 1995). Types I to III have been studied extensively (Gunnaiah et al. 2012). Some resistant genotypes, such as Nobeokabouzu-komugi (Type I resistance), Sumai-3 and Wangshuibai (Type II resistance), and Frontana (Type III resistance) have been identified (Niwa et al. 2014); however, highly resistant germplasm has not been observed to date. Moreover, the same variety sourced from various geographical

${ }^{\dagger}$ Corresponding author: R. N. Chibbar; E-mail: ravi.chibbar@usask.ca

P. Sharma and M. P. Gangola contributed equally to the work.

(C) 2018 The American Phytopathological Society locations has high genomic diversity and significant differences in FHB resistance. For example, Sumai-3 from Austria (Sum-Aut) and Japan (Sum-Jap) had different FHB symptom scores (1.0 to 2.5 and 3.0 to 4.7 at 4 weeks after inoculation), as well as differences in the ratio of FHB-damaged kernels (22.5 to 24.2 and 47.3 to 61.9) in field experiments in 2009 and 2010 (Niwa et al. 2014). Therefore, new stable genetic resources are needed that can be utilized in breeding programs to develop FHB-resistant wheat varieties. One of the methods to generate such new genetic resources is through chemical or physical mutagenesis. Among the chemical mutagens, ethyl methane sulfonate (EMS) is the most widely used in plants; it induces random point mutations (Talebi et al. 2012). The mutagenized plants can be evaluated for FHB resistance and mutations associated to FHB-resistant genes reported previously.

FHB resistance in wheat is a quantitative trait (Snijders 2004). Among the more than 100 quantitative trait loci (QTL) reported for FHB resistance in wheat, Qfhs.ndsu-3BS or Fhbl is the most repeatable. It is on the $3 \mathrm{BS}$ chromosome and has been reported in at least 26 different studies (Buerstmayr et al. 2009; Liu et al. 2006; Waldron et al. 1999). Recently, mutation, gene silencing, and transgenic overexpression studies identified a pore-forming toxin (PFT) gene as a candidate for Fhbl (Rawat et al. 2016). Wheat lines with Fhbl exhibit a greater deoxynivalenol-3-O-glucoside:deoxynivalenol (D3G:DON) ratio than lines without Fhbl (Rawat et al. 2016), implying that the metabolism of DON plays a significant role in FHB resistance (Lemmens et al. 2005). The detoxification of DON to D3G was associated with UDP-glucosyltransferase (UGT) genes as they were present on the same chromosome (Rawat et al. 2016). The members of the UDP-glucosyltransferase (UGT) gene family encode UDP-glucosyltransferases (EC 2.4.1.x) that catalyze the transfer of glucose from UDP-glucose to the hydroxyl group at carbon-3 of deoxynivalenol leading to production of nontoxic DON-3O-glucoside (D3G) (Wetterhorn et al. 2016). In wheat, several UGTs (GenBank accessions EU568801.1, FJ236328.1, and FG985273.1) have been reported, but their validation in a population varying for FHB resistance has not been studied. 
In vivo methods are commonly used to assess FHB disease severity. Since not all varieties and plants of the same variety flower simultaneously, some experimental variation between early and late flowering plants may exist. Moreover, disease scoring is time consuming and inconsistent as in vivo methods are subject to environmental conditions (Shin et al. 2014). Therefore, a rapid, uniform, reliable and laboratory-based method is required for FHB screening. In the present study, the in vitro spike culture technique (Ganeshan et al. 2010) was optimized to screen for FHB resistance in wheat genotypes derived from a spike culture-derived variant (SCDV) creation strategy using EMS (Ganeshan and Chibbar 2017). One of the potential candidate target genes for FHB resistance, UDP-glucosyltransferase (TaUGT) was analyzed by DNA sequencing in control, resistant, and susceptible genotypes to identify single nucleotide polymorphisms (SNPs) associated with FHB resistance. High resolution melt (HRM) curve analysis for SNP detection revealed a significant correlation of B-genome specific TaUGT-2/TaUGT-3 to FHB severity and reduced DON accumulation in wheat kernels from FHB-resistant SCDV lines.

\section{MATERIALS AND METHODS}

Development of SCDV population. The spike culture system was used to generate an EMS-mutagenized population of wheat, referred to as SCDV (Ganeshan and Chibbar 2017). Briefly, immature spikes of wheat cultivar AC Nanda (Sadasivaiah et al. 2000) were treated with EMS at a concentration of $0.25 \%$ (wt/vol) for $4 \mathrm{~h}$ to generate the initial $\mathrm{M}_{0}$ population. The seeds were germinated to produce $\mathrm{M}_{0}$ plants, of which each head was maintained separately. $M_{0}$ plants were self-fertilized to produce the $M_{1}$ seeds. The $M_{2}$ seeds from each spike of $\mathrm{M}_{1}$ plant were also maintained separately. As the EMS treatment was applied to the culture medium prior to anthesis, the $\mathrm{M}_{2}$ population was expected to be less heterozygous. Three $\mathrm{M}_{2}$ seeds from three spikes were planted to grow $\mathrm{M}_{2}$ plants. For DNA extraction and SNP analyses, leaf samples were collected from 20-day-old plants. The immature spikes were screened for FHB resistance.

Seeds of Triticum monococcum L. (CN 37598), Aegilops speltoides Tausch (CN 45194), and Aegilops tauschii Coss. (CN 30812) were procured from Plant Gene Resources of Canada (PGRC, Saskatoon, SK, Canada).

Optimization and utilization of spike culture as a tool to screen FHB resistance in the SCDV population. Soft white spring wheat plants (cultivar AC Nanda) were grown in pots containing Sunshine Professional growing mix (Sun Gro Horticulture Canada Ltd., Agawam, MA) in a growth chamber at $20^{\circ} \mathrm{C} / 16 \mathrm{~h}$ light $\left(350 \mu \mathrm{mol} / \mathrm{m}^{2} / \mathrm{s}\right.$ PPFD) and $18^{\circ} \mathrm{C} / 8 \mathrm{~h}$ dark. Plants were fertilized every 3 weeks with slow release fertilizer, Nutricote-14: 14:14-N:P:K (Plant Products Co. Ltd., Brampton, ON, Canada). Main tillers bearing immature spikes were cut above the soil surface on the day spikes fully emerged (Feekes scale 10.5; Haun scale 11). After removing leaves, the spikes were placed in tubes containing $5 \mathrm{ml}$ of culture medium (pH 6.2) consisting of sucrose at $50 \mathrm{~g} / \mathrm{liter}$ and L-glutamine at $0.4 \mathrm{~g} /$ liter, buffered with morpholinoethanesulfonic acid at $0.5 \mathrm{~g} /$ liter (Ganeshan and Chibbar 2014). The spikes were transferred to fresh culture medium every 3 days and point inoculated with $F$. graminearum after $0,2,5,8,11$, and 14 days in the culture media.

Four Fusarium graminearum sensu stricto (s.s.) isolates FGSC9075, M1-07-2, M7-07-1, and M9-07-1 (kindly provided by Cereal Research Centre, Agriculture and Agri-Food Canada, Winnipeg) were inoculated into spikes. Isolate FGSC 9075 is a wild-type Fusarium for which full genomic information is available. FGSC9075 and M1-07-2 are 15-acetyl-deoxynivalenol (15-ADON) chemotypes, whereas M9-07-1 and M7-07-1 are 3-ADON chemotypes. Macroconidial suspensions of isolates were prepared using 7-day-old potato dextrose agar (PDA) cultures. Macroconidia were dislodged in $5 \mathrm{ml}$ of sterile distilled water with a sterile glass rod. Suspensions were filtered through four layers of cheese cloth to remove mycelium and agar pieces. Macroconidial concentrations were determined microscopically using a hemocytometer and were adjusted to $10^{6}$ macroconidia/ml using sterile distilled water. Serial dilutions were then made to achieve concentrations of $10^{5}, 10^{4}$, and $10^{3}$ macroconidia/ml. Final concentrations were also confirmed by microscopic counts using a hemocytometer.

To determine inoculum viability, $15 \mu \mathrm{l}$ of inoculum was plated on water-agar media. In all cases, $>99 \%$ of macroconidia germinated. Tween $20(0.02 \%, \mathrm{vol} / \mathrm{vol})$, a nonionic surfactant, was added as a wetting agent to prepare the conidial suspensions. The macroconidial suspensions were injected between the lemma and palea of a basal floret positioned in the middle of the spikes. Control spikes were inoculated with sterile distilled water with $0.02 \%$ Tween-20 (vol/vol). To maintain humidity and promote infection, the inoculated spikes were misted with distilled water and covered with plastic bags for three days. The infected spikes were placed in Sanyo Versatile Environmental Test Chamber (Sanyo MIR-351H, Canada) at $16 \mathrm{~h}$ days $\left(26^{\circ} \mathrm{C}\right)$ and $8 \mathrm{~h}$ nights $\left(24^{\circ} \mathrm{C}\right)$. After 3 days, bags were removed and disease developed. From 5 days of inoculation, spikes were rated at every second day for disease symptoms until bleaching was observed due to natural senescence (aging). The numbers of bleached and total spikelets were counted for each treated spike in culture to calculate disease severity (number of diseased spikelets divided by the total number of spikelets). All experiments were repeated three times. The optimized protocol of in vitro spike culture techniques was used to screen the SCDV population for FHB resistance.

SNP identification in TaUGTs. Genomic DNA from wheat leaves was extracted following the manufacturer's protocol (Qiagen DNeasy Plant Mini Kit, Qiagen, Germantown, MD). Oligonucleotide primers (Table 1) were designed to amplify the UDP-glucosyl transferases (UGT): TaUGT-2 (GenBank accession EU568801.1), TaUGT-3 (GenBank accession FJ236328.1), and TaUGT-EST (GenBank accession FG985273.1). The primers were first assessed in a PCR reaction for their genome specificity using DNA from T. monococcum (2A genome), A. speltoides (2B genome), A. tauschii (2D genome), AC Nanda (2A2B2D), and Sumai-3 (2A2B2D). The controls were AC Nanda (susceptible) and Sumai-3 (resistant). The corresponding PCR amplified products were gel eluted (AccuPrep gel purification kit, Bioneer Inc., Daejeon, Republic of Korea) and cloned in the pJET1.2/blunt cloning vector (Fermentas Canada Inc., Burlington, ON, Canada). For each gene, plasmids isolated from nine clones from three independent PCR reactions were DNA sequenced for AC Nanda and Sumai-3 using BIGDYE Terminator v3.1 (Applied Biosystems Inc., CA) at Eurofins MWG Operon (Huntsville, $\mathrm{AL}$ ). The nucleotide sequences were analyzed for similarity with the publicly available nonredundant genes/transcripts in the database (https://www.ncbi.nlm.nih.gov/BLAST) using the BLASTN algorithms. Multiple sequence alignment was executed to find nucleotide variants or SNP in FHB-resistant and -susceptible wheat genotypes using CLC Genomics Workbench 4 software (CLC Bio, Cambridge, MA).

SNP validation in the SCDV population using HRM and DNA sequencing. For mutation/SNP screening, an HRM assay was performed using genomic DNA extracted from leaves of $\mathbf{M}_{2}$ plants. Nested PCR was used to detect mutations in the B-genome specific TaUGT-2 and TaUGT-3: (i) B-genome specific sequences of TaUGT-2 and TaUGT-3 were amplified from genomic DNA using TaUGT-2F1/2R2 and TaUGT-3F1/3R2 (Table 1), respectively; (ii) primers targeting a specific SNP in TaUGT-2 (at $450 \mathrm{bp}$ from the start codon) and TaUGT-3 (at 1,558 bp from the start codon) were designed for the HRM assay. AC Nanda and Sumai-3 differed at these mutation sites (Table 1). D-genome specific primers (TaUGTHF3/HR3) were used for the HRM assay of TaUGT-EST using genomic DNA (Table 1).

The $20-\mu \mathrm{l}$ reaction volume for the HRM assay included $10 \mu \mathrm{l}$ of MeltDoctor HRM Master Mix (Applied Biosystems, Inc., CA), $0.3 \mu \mathrm{M}$ of each primer, $1 \mu \mathrm{l}$ of PCR product as DNA template and 
sterile deionized water. No template controls were included in PCR assays. The HRM assay was performed in two consecutive steps, amplification and melt curve analysis, using a 7500 Fast Real-Time PCR system (Applied Biosystems). The amplification step consisted of an initial denaturation at $95^{\circ} \mathrm{C}$ for $10 \mathrm{~min}$, followed by 40 cycles of $95^{\circ} \mathrm{C}$ for $15 \mathrm{~s}$ and $60^{\circ} \mathrm{C}$ for $30 \mathrm{~s}$. The HRM step comprised of denaturation at $95^{\circ} \mathrm{C}$ for $10 \mathrm{~s}, 60^{\circ} \mathrm{C}$ for $1 \mathrm{~min}$ (annealing), $95^{\circ} \mathrm{C}$ for $15 \mathrm{~s}$ (HRM) and a final annealing step at $60^{\circ} \mathrm{C}$ for $1 \mathrm{~min}$. The step from $60^{\circ} \mathrm{C}$ to $95^{\circ} \mathrm{C}$ was carried out in steps of $0.1^{\circ} \mathrm{C}$, each step with a $10 \mathrm{~s}$ hold. The high-resolution melt curve profile was analyzed using HRM analysis software version 2.0.1 (Applied Biosystems). The EMS variants with nucleotide variation were easily distinguished by plotting the fluorescence differences among melt curves. The HRM results were validated by DNA sequencing of more than $50 \%$ of the SCDV population, i.e., 70 independent PCR products, including AC Nanda and Sumai-3 and 68 variants.

Statistical analysis. Frequency distribution graphs and box plots were prepared using MS-Excel (MS Office Professional Plus 2013, Microsoft Corporation, CA). One-way analyses of variance (ANOVA) and pairwise comparisons (Tukey's method of Post hoc analysis) were performed using Minitab software 16 (Minitab, Inc., Pennsylvania).

Semiquantitative expression analysis. Semiquantitative reverse transcription-PCR (RT-PCR) analysis was performed to study the transcript abundance of TaUGT-2 and 3 during FHB progression in wheat spikes. FHB-resistant (250.2, 202.5, and 248.1) and susceptible $(68.4,123.5$, and 136.6) SCDVs (identified in the present study), AC Nanda (FHB-susceptible control), and Sumai-3 (FHB-resistant control) were grown during January to March 2015 in a greenhouse (University of Saskatchewan, Canada) with $18 \mathrm{~h}$ photoperiod, $240.5 \mu \mathrm{mol} / \mathrm{m}^{2} / \mathrm{s}$ of integrated PAR (photosynthetically active radiation), at a mean temperature from $20^{\circ} \mathrm{C}$ (night) to $21.7^{\circ} \mathrm{C}$ (day) and approximately $33 \%$ relative humidity. The immature spikes were collected, cultured in spike culture medium and point inoculated with $F$. graminearum strain M7-07-01 as described previously. The inoculated spikes were collected from immediately after inoculation up to 8 days after inoculation (DAI) at 2-day intervals. The harvested samples were immediately frozen in liquid nitrogen and stored at $-80^{\circ} \mathrm{C}$. Oligonucleotide primers (Table 1) for gene expression analyses were designed from the sequences of cloned genomic DNA (Table 1). The RNA was extracted and pooled from three spikes $(250 \mathrm{mg})$ using the manufacturer's protocol (PureLink RNA Mini Kit Catalogue number
12183018A, Ambion by Life Technologies, Carlsbad, CA). The RNA $(5 \mu \mathrm{g})$ was treated with DNase (TURBO DNA-free Kit, Ambion by Life Technologies, Carlsbad, CA) and first strand cDNA was synthesized as described in the product manual (SuperScript IV First-Strand Synthesis System, Catalog number: 18091050, Invitrogen by Life Technologies, Carlsbad, CA). The resulting cDNA from various stages of the FHB inoculated wheat spikes were used to study $U G T$ expression. A PCR reaction of $25 \mu \mathrm{l}$ included $2.5 \mu \mathrm{l}$ of $10 \times$ DreamTaq buffer, $2 \mathrm{mM} \mathrm{MgCl} 2,0.2 \mathrm{mM}$ of dNTPs, $0.2 \mu \mathrm{M}$ of forward and reverse primers (Table 1), $0.625 \mathrm{U}$ of DreamTaq DNA polymerase, nuclease free water (as needed), and cDNA $(0.5 \mu \mathrm{l}$ of TaUGT-2B or $1.0 \mu \mathrm{l}$ of TaUGT-3B). The PCR conditions for TaUGT-2B, TaUGT-3B, and TaUGT-EST were optimized as $95^{\circ} \mathrm{C}$ for $10 \mathrm{~min}, 30 / 32$ (TaUGT-2B and TaUGT-EST/TaUGT-3B) cycles of $95^{\circ} \mathrm{C}$ for $1 \mathrm{~min}, 60^{\circ} \mathrm{C}$ for $0.5 \mathrm{~min}$ and $72^{\circ} \mathrm{C}$ for $0.5 \mathrm{~min}$, followed by $72^{\circ} \mathrm{C}$ for $10 \mathrm{~min}$. The wheat $R P L S 8$ (ribosomal protein S8) gene (Table 1) was used as a reference gene (Ganeshan et al. 2012; Sharma et al. 2013).

DON determination in FHB-infected spikes. Wheat spikes, collected for semiquantitative expression analysis, were also utilized to determine DON concentration by a competitive colorimetric ELISA assay (AgraQuant Deoxynivalenol Assay 0.25/5.0, Romer Labs Singapore Pte. Ltd., Singapore). The DON was extracted and its concentration was determined following the manufacturer's protocol. The limit of detection was $0.2 \mathrm{ppm}$ and the quantification of the assay $0.25 \mathrm{ppm}$, whereas the range of quantification was from 0.25 to $5.0 \mathrm{ppm}$. Samples with $>5 \mathrm{ppm}$ DON concentration were diluted and reanalyzed to obtain accurate results.

\section{RESULTS}

Optimization of in vitro spike culture technique. The following important steps of the spike culture and point inoculation methods were optimized: (i) days of growing spikes in the culture media, (ii) selection of pathogen isolate, and (iii) concentration of pathogen inoculum. Considered over the range of $0,2,5,8,11$, and 14 days in culture media, wheat spikes grown for 5 days were found suitable for pathogen inoculation and FHB resistance screening. Depending on the isolates and wheat (AC Nanda) spike stage after heading in culture media, inoculum concentrations of $10^{3}, 10^{4}, 10^{5}$, and $10^{6}$ macroconidia/ml caused 9 to $19 \%, 10$ to $31 \%, 19$ to $66 \%$, and 38 to $87 \%$ disease severity, respectively, 7 days after inoculation

TABLE 1. Oligonucleotide primer sequences used for gene isolation, high resolution melt (HRM) curve analysis, and transcript accumulation during Fusarium head blight infection in wheat spikes

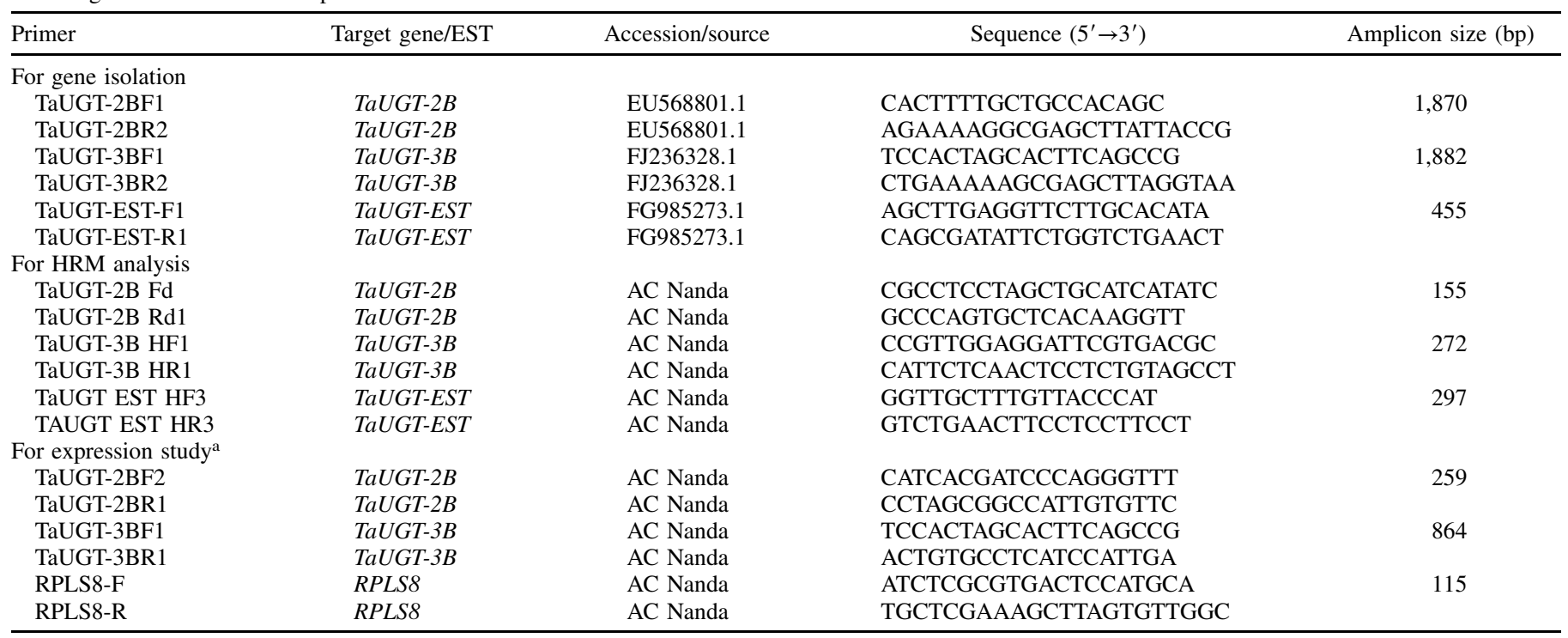

a Amplicon sizes correspond to cDNA sequences. 
(Fig. 1). ANOVA revealed that inoculum concentration affected disease severity, whereas the number of days after heading at which inoculum was administered did not. Results showed that $10^{5}$ macroconidia/ml was the most appropriate concentration to screen FHB resistance compared with $10^{3}, 10^{4}$, and $10^{6}$ macroconidia $/ \mathrm{ml}$ (Fig. 1). The concentrations of $10^{3}$ and $10^{4}$ macroconidia/ml overestimated moderate FHB resistance, whereas disease severity observed at $10^{6}$ macroconidia/ml overwhelmed moderate FHB resistance. Fusarium isolates also affected disease severity but only at $10^{5}$ macroconidia/ml. In the spike culture system, isolate M7-071 was moderately virulent and therefore used to screen for FHB resistance. However, M9-07-1 was found to be the most virulent, followed by M7-07-1, M1-07-2, and FGSC-9075.

Variation in FHB severity among SCDV. The SCDV population (134 wheat variants) along with AC Nanda and Sumai-3 differed for FHB resistance. For AC Nanda, disease severity was $54 \%$, whereas Sumai-3 had disease severity of approximately $35 \%$. The frequency graph of SCDV for FHB severity had a bimodal distribution ranging from 15 to $100 \%$ (Fig. 2). There was no variant with complete FHB resistance. Approximately $51 \%$ of the lines had a disease severity of 0 to $40 \%$, whereas the remaining $49 \%$ were between 41 to $100 \%$ at 7 DAI. All the disease severity values were recorded at 7 DAI. The SCDV lines 250.2, 202.5, and 248.1 were identified to have the most promising FHB resistance, and SCDV lines 68.4, 123.5, and 136.6 were deemed to be susceptible.

Identifying SNPs in UGTs and screening of SCDVs for their presence. Gene specific primers (TaUGT2F1/2R2 and TaUGT3F1/3R2) for TaUGT-2 and TaUGT-3 amplified PCR products using DNA extracted from $A$. speltoides (B genome) and AC Nanda. The amplicon size was 1,870 bp for TaUGT-2 and 1,882 bp for TaUGT-3. No PCR products were obtained with DNA from T. monococcum (A genome) or A. tauschii (D genome) (Fig. 3). Therefore, TaUGT-2 and TaUGT-3 were renamed as TaUGT-2B and TaUGT-3B to emphasize their B genome specificity. However, primers for TaUGT-EST amplified a 455-bp product from
T. monococcum, A. speltoides, A. tauschii, and AC Nanda (Fig. 3). The amplicons were cloned, DNA sequenced using nested primers, and subsequently confirmed by BLAST (Altschul et al. 1990). The TaUGT-2B and TaUGT-3B nucleotide sequences were approximately 99 and 97\% identical with accession numbers ACB47288.1 and EU552210.1, whereas UGT-EST was approximately 96 and $89 \%$ similar to UGT from A. tauschii (D genome; EMT21930.1) and T. urartu (A genome; EMS46301.1)/T. aestivum (afx95778.1), respectively.

The amplified genomic DNA sequence of TaUGT-2B was $1,870 \mathrm{bp}$ long with an open reading frame (ORF) of $1,815 \mathrm{bp}$ including two exons (494 and $997 \mathrm{bp}$ ) separated by one intron (324 bp). The TaUGT-2B ORF was flanked by 27 and 28 bp of the $5^{\prime}$ and $3^{\prime}$ untranslated region (UTR). The 1,882 bp of TaUGT-3B amplified was composed of two exons (497 and $994 \mathrm{bp}$ ) separated by one intron (337 bp) and flanked by a $26 / 28$ bp as the $5^{\prime} / 3^{\prime}$ UTR (Fig. 4). Analysis of AC Nanda and Sumai-3 gene sequences

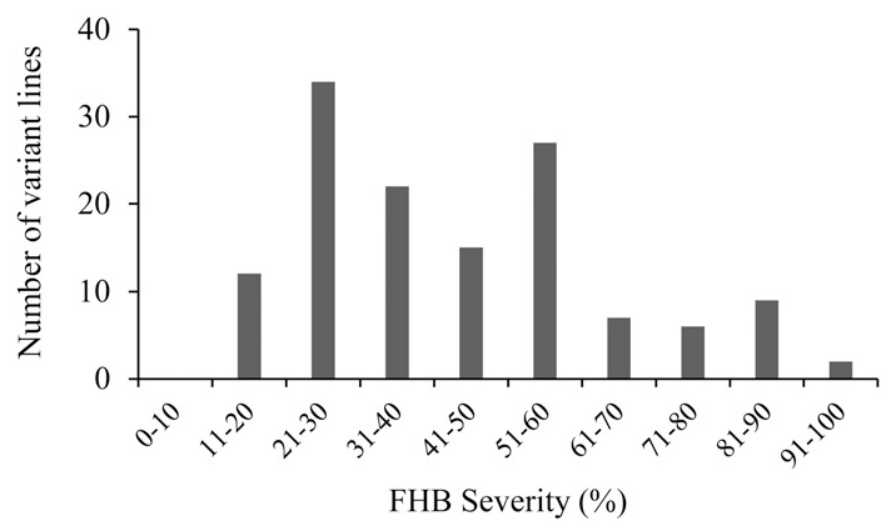

Fig. 2. Frequency distribution of wheat spike culture-derived variants (SCDV) indicating the range of Fusarium head blight resistance.
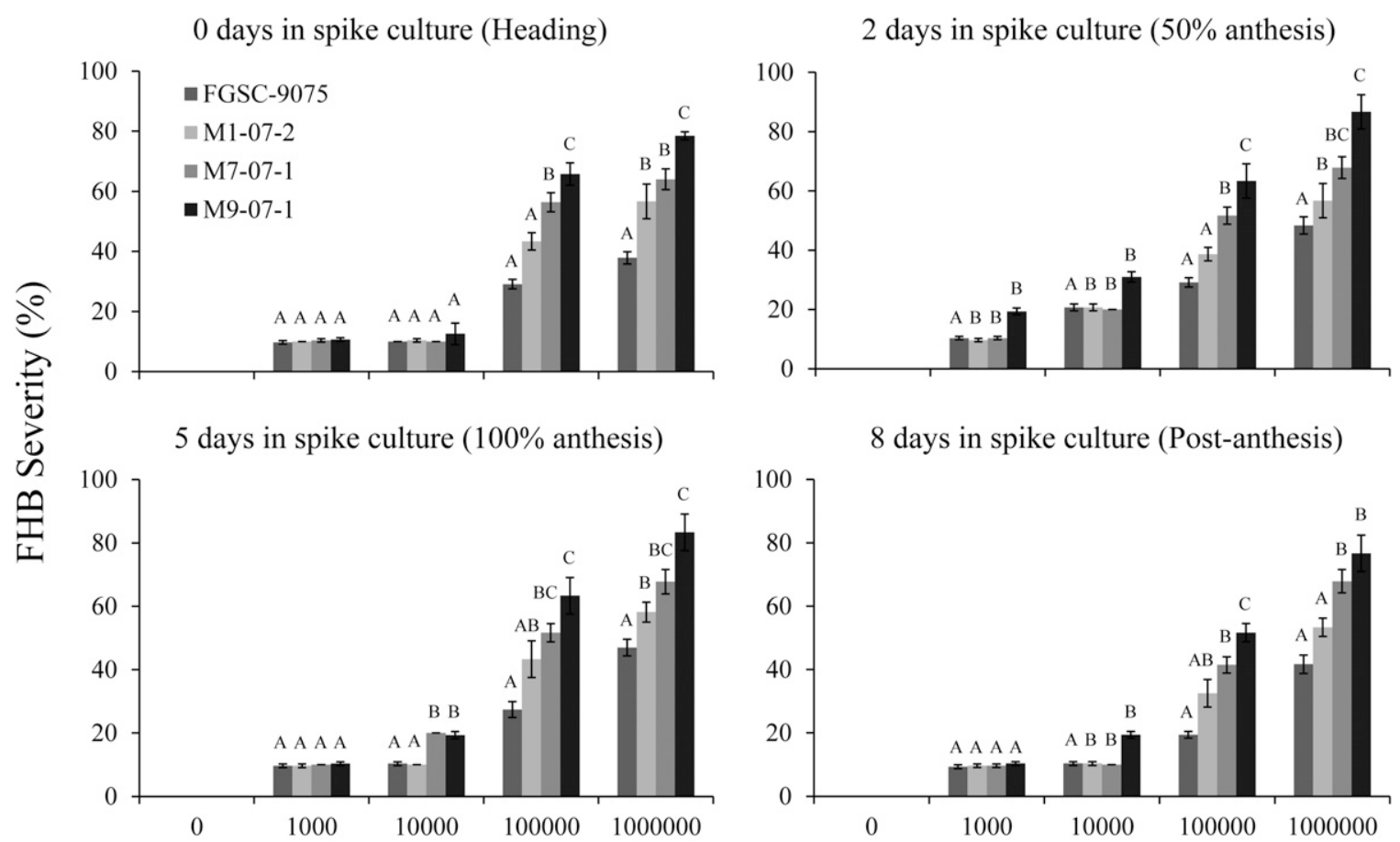

\section{Number of macroconidia/ml}

Fig. 1. Effect of increasing concentrations of Fusarium graminearum isolates FGSC-9075, M1-07-2, M7-07-1, and M9-07-1 on Fusarium head blight disease severity in wheat cultivar AC Nanda spikes cultured for 0,2,5, and 8 days. Values are means \pm standard deviations based on three independent determinations. Values followed by different letters show significant differences. 
revealed one SNP in both TaUGT-2B $[450 \mathrm{bp}(\mathrm{A} \rightarrow \mathrm{G})]$ and TaUGT$3 \mathrm{~B}[1,558 \mathrm{bp}(\mathrm{G} \rightarrow \mathrm{T})]$, from the start codon in exon 1 and 2 , respectively (Fig. 4). A SNP in UGT-EST was detected at position 229 bp between AC Nanda (G) and Sumai-3 (A) sequence that incorporated an early stop codon in Sumai-3 (Fig. 4).

For mutation screening of SCDV, an HRM curve assay was done. Nested PCR amplified DNA specifically from TaUGT- $2 B$ and TaUGT-3B. Analysis of fluorescent signals detected differences between individual plants that varied in nucleotide sequence. The differences between TaUGT-2B and TaUGT-3B resulted in two distinct groups, one included AC Nanda and the other Sumai-3 (Fig. 5). At $450 \mathrm{bp}$ from the translation initiation site in TaUGT-2B,34 SCDVs had nucleotide 'A' similar to AC Nanda, whereas 100 SCDVs had nucleotide ' $G$ ', similar to Sumai-3. Similarly, for TaUGT-3B, 29 SCDV had nucleotide ' $G$ ' at position 1,558 bp as did AC Nanda, whereas 105 SCDV had ' $T$ ' as in Sumai-3 (Table 2). To confirm these results, independent gel eluted PCR products of TaUGT-2B from randomly selected 68 SCDV were DNA sequenced, which validated the HRM

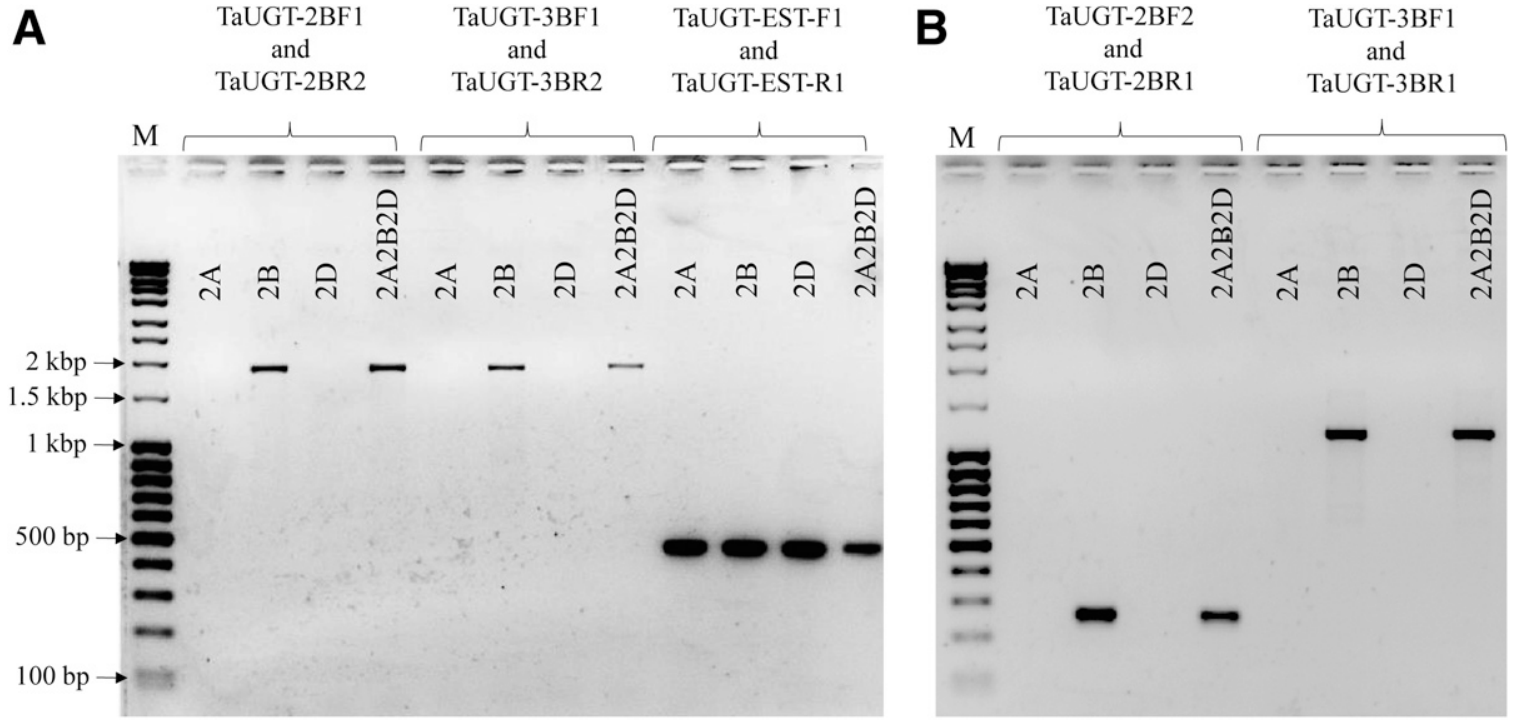

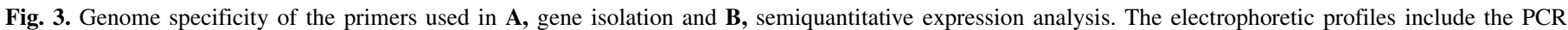
products amplified from Triticum monococcum (2A), Aegilops speltoides (2B), Aegilops tauschii (2D), and Triticum aestivum 'AC Nanda' (2A2B2D).

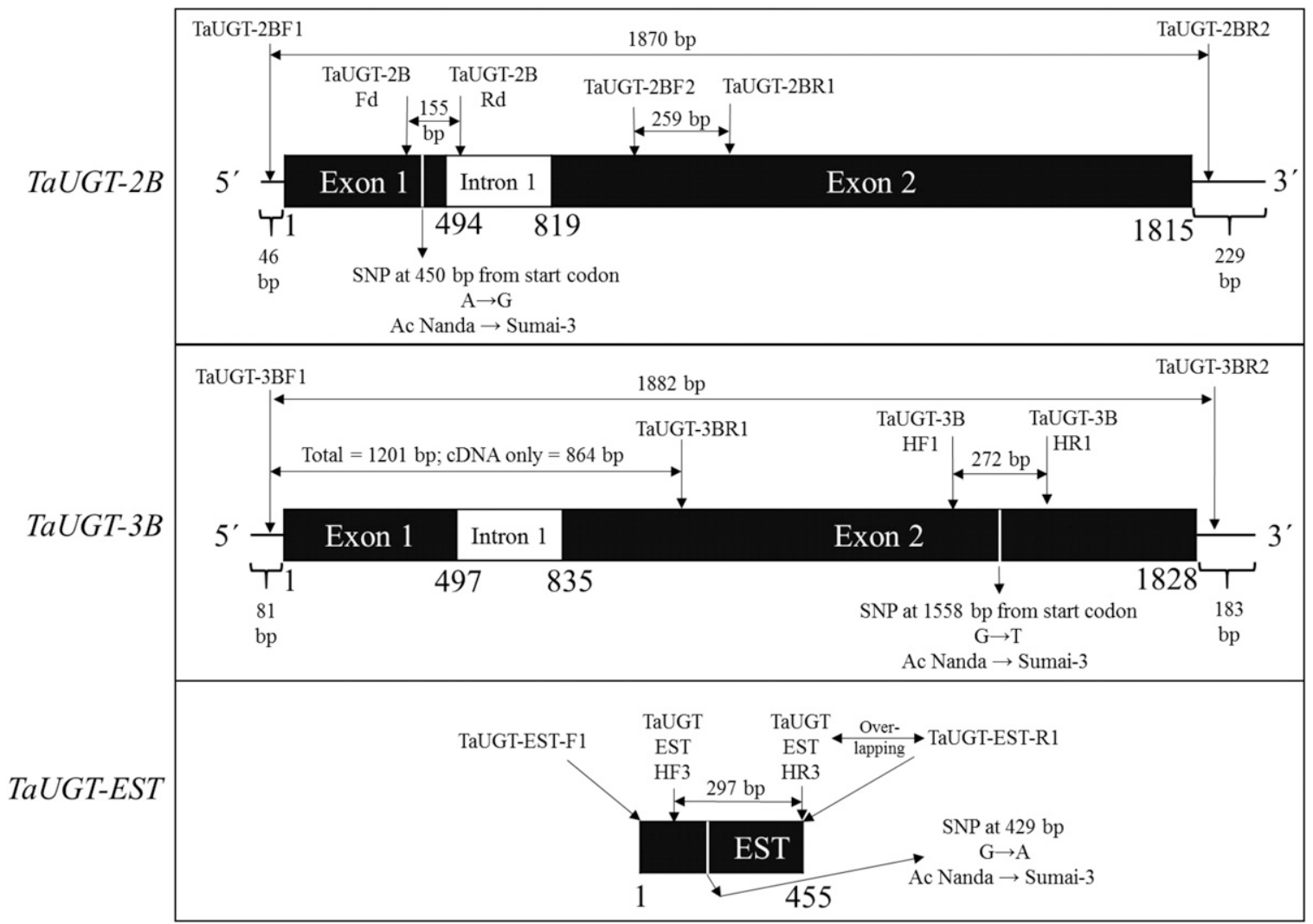

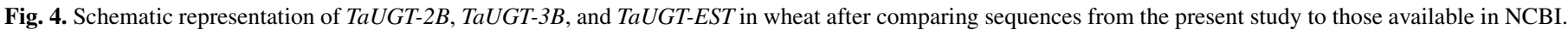
The sequences show primer sites, amplicon length, location of single nucleotide polymorphisms (SNP), and distribution of exons/introns/untranslated regions. 
results (Fig. 5). The D-genome specific primer of TaUGT-EST, however, produced only one cluster containing AC Nanda and all SCDVs. Only Sumai-3 differed as was expected due to a nucleotide difference at 229 bp (Fig. 5).

One-way ANOVA revealed an effect of SNPs at position $450 \mathrm{bp}$ in TaUGT-2B and 1,558 bp in TaUGT-3B on FHB disease severity (Table 2). Boxplot analysis together with pairwise comparisons using Tukey's test revealed differences between groups (Fig. 6). The two groups for TaUGT-2B SNP had FHB severities of 55\% for those with 'A' and $40 \%$ for those with ' $G$ ', whereas the mean FHB severity for the two groups of TaUGT-3B were $55 \%$ for ' $\mathrm{G}$ ' and $41 \%$ for ' $\mathrm{T}$ '. Based on nucleotide sequence variation and the mean value of FHB disease severity at 7 and 9 days after inoculation, the 15 most promising SCDV genotypes were selected. These may be of value in breeding programs (Table 3 ).

Semiquantitative expression analysis. The amplicon size in TaUGT-2B was 259 bp and in TaUGT-3B, 864 bp (Fig. 3). In FHBsusceptible genotypes, both TaUGT-2B and TaUGT-3B had little expression during initial stages of FHB infection. However, the expression of both genes was induced mainly at 6 DAI. TaUGT-2B and TaUGT-3B expression was induced early at 2 DAI in all FHBresistant lines and decreased gradually toward maturity (Fig. 7). TaUGT-EST followed a similar expression pattern to TaUGT-2B and TaUGT-3B in all genotypes except 123.4 and Sumai-3, whereas its expression was very low in 136.6. In 123.4, expression of TaUGTEST was induced at 2 DAI, while in Sumai-3 expression increased and decreased in a random manner.

Determination of deoxynivalenol (DON) concentration. The $\log / \log$ it regression model was used to prepare a calibration curve (Fig. 8A) and determine DON concentration in wheat (Fig. 8B). The linearity coefficient $\left(R^{2}\right)$ of the calibration curve was -0.993 . DON was either absent or at a trace immediately after inoculation (0 DAI) in all wheat genotypes. The maximum DON accumulation at
2 DAI in FHB-resistant SCDVs ranged from 1,211 (SCDV 202.6) to 2,117 (SCDV 248.1) ppm, which decreased gradually thereafter. At 8 DAI, DON concentration varied from $498 \mathrm{ppm}$ (SCDV 202.6) to 988 ppm (SCDV 250.2). In FHB-susceptible lines, DON accumulated until 8 DAI, at which time it was the maximum concentration and varied from 2,153 to $2,980 \mathrm{ppm}$.

\section{DISCUSSION}

In vitro spike culture technique is a rapid and reliable method to screen for FHB resistance. To overcome the complex, time-consuming, and environmentally dependent challenges of field-based FHB screening (Parry et al. 1995; Shin et al. 2014), an in vitro spike culture technique (Ganeshan et al. 2010) was optimized for FHB resistance screening as a prerequisite of this study. Natural senescence started in control wheat spikes after 15 days in spike culture media; therefore, it was not possible to distinguish between bleaching of spikelets due to pathogen inoculation and natural senescence. Inoculated spikes took about 5 days to develop FHB symptoms. Disease scoring began at $5 \mathrm{DAI}$, the first stage at which FHB symptoms were detected and continued until $11 \mathrm{DAI}$ at 2-day

TABLE 2. One-way analysis of variance indicating significant effects of single nucleotide polymorphisms in B-genome specific UDP-glucosyl transferases on Fusarium head blight (FHB) disease severity

\begin{tabular}{lccccc}
\hline Gene & $\begin{array}{c}\text { Nucleotide } \\
\text { position }\end{array}$ & Allele & $\begin{array}{c}\text { Number of } \\
\text { variant } \\
\text { lines }\end{array}$ & $\begin{array}{c}\text { Mean sum of } \\
\text { squares for } \\
\text { FHB severity }\end{array}$ & $\begin{array}{c}\text { Significant } \\
\text { at } P\end{array}$ \\
\hline TaUGT-2B & 450 & $\mathrm{~A}$ & 34 & 6,129 & $\leq 0.001$ \\
TaUGT-3B & 1,558 & $\mathrm{G}$ & 100 & & $\leq 0.001$ \\
& & $\mathrm{G}$ & 29 & 4,458 & \\
\hline
\end{tabular}

\section{Aligned melt curves}

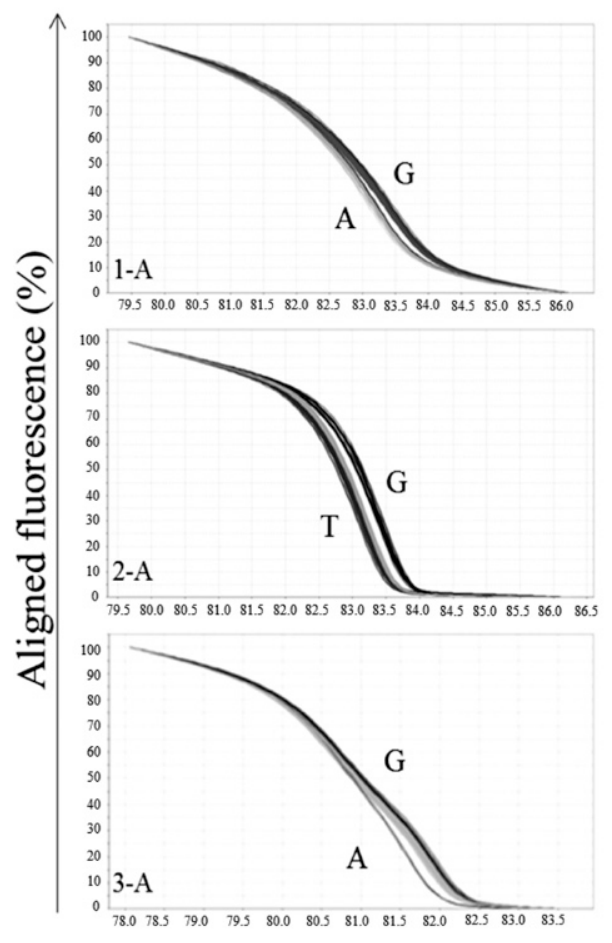

\section{Difference plot}

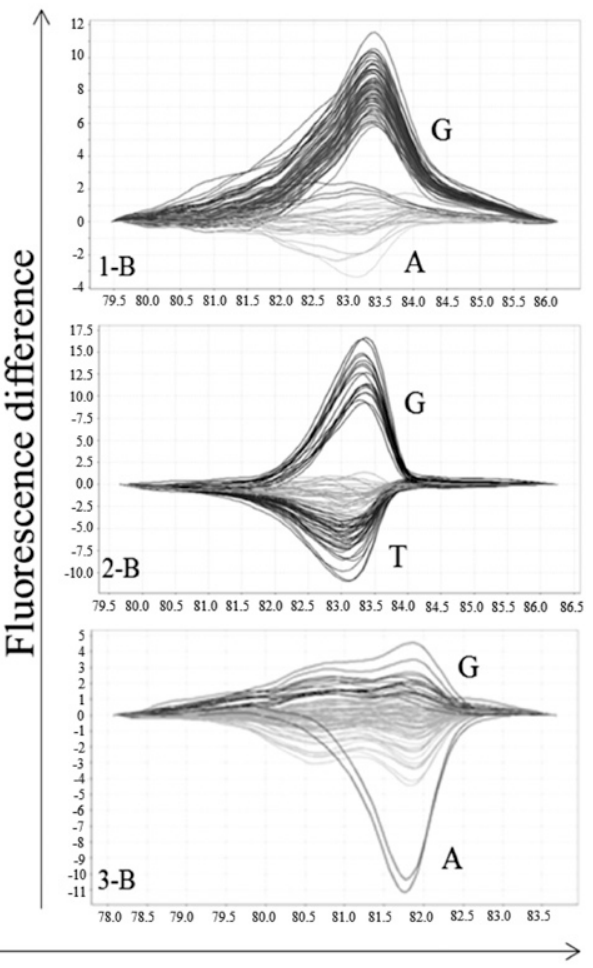

Temperature $\left({ }^{\circ} \mathrm{C}\right)$

Fig. 5. High resolution melt curve (HRM) analysis with primers TaUGT-2B Fd/Rd-1, TaUGT-3BHF1/HR1, and TaUGT-EST HF3HR3 in terms of aligned fluorescence $(\mathrm{F}, \%)$ versus temperature $(\mathrm{T})$ curves, and $\Delta \mathrm{F} / \mathrm{T}$ difference curves separating Fusarium head blight resistant and susceptible genotypes into two distinct groups ( $1 \mathrm{~A}$ and $1 \mathrm{~B}$ and $2 \mathrm{~A}$ and $2 \mathrm{~B})$. In $3 \mathrm{~A}$ and $3 \mathrm{~B}$, only Sumai-3 was detected with single nucleotide polymorphisms. 
intervals. After 5 days in culture, spikes were at an appropriate stage for inoculation because of the following: (i) spikes were well established in the culture media, (ii) FHB disease screening could be completed before the start of natural senescence, and (iii) it

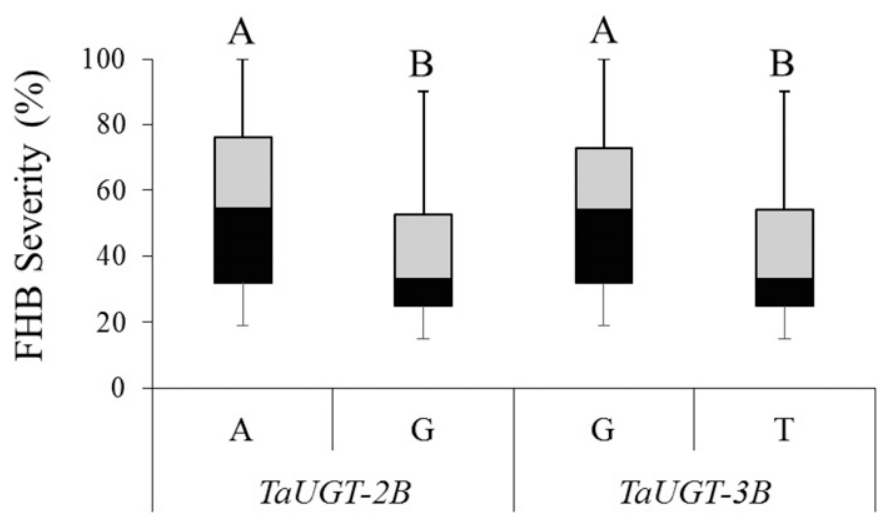

Fig. 6. Box plot analysis for TaUGT-2B and TaUGT-3B indicating the significant association of single nucleotide polymorphisms with Fusarium head blight disease severity. Values followed by different letters indicate significant differences.

TABLE 3. Fifteen of the most promising spike culture-derived variants (SCDV) that may be used in a wheat breeding program to develop Fusarium head blight (FHB) resistant varieties

\begin{tabular}{|c|c|c|c|c|}
\hline \multirow[b]{2}{*}{ SCDV } & \multirow{2}{*}{$\frac{\text { TaUGT2-B }}{450 \mathrm{bp}}$} & \multirow{2}{*}{$\frac{\text { TaUGT3-B }}{1,555 \mathrm{bp}}$} & \multicolumn{2}{|c|}{ FHB severity $(\%)$} \\
\hline & & & $7 \mathrm{DAI}^{\mathrm{a}}$ & 9 DAI \\
\hline 250.2 & $\mathrm{G}$ & $\mathrm{T}$ & 15 & 23 \\
\hline 66.4 & $\mathrm{G}$ & $\mathrm{T}$ & 17 & 26 \\
\hline 250.3 & G & $\mathrm{T}$ & 27 & 41 \\
\hline 248.1 & $\mathrm{G}$ & $\mathrm{T}$ & 20 & 24 \\
\hline 81.5 & G & $\mathrm{T}$ & 23 & 23 \\
\hline 223.6 & G & $\mathrm{T}$ & 24 & 24 \\
\hline 98.6 & $\mathrm{G}$ & $\mathrm{T}$ & 21 & 29 \\
\hline 105.6 & $\mathrm{G}$ & $\mathrm{T}$ & 21 & 29 \\
\hline 202.6 & $\mathrm{G}$ & $\mathrm{T}$ & 21 & 33 \\
\hline 246.4 & $\mathrm{G}$ & $\mathrm{T}$ & 21 & 33 \\
\hline 213.4 & $\mathrm{G}$ & $\mathrm{T}$ & 23 & 32 \\
\hline 234.5 & $\mathrm{G}$ & $\mathrm{T}$ & 25 & 30 \\
\hline 247.1 & G & $\mathrm{T}$ & 19 & 38 \\
\hline 192.6 & G & $\mathrm{T}$ & 25 & 33 \\
\hline 202.5 & G & $\mathrm{T}$ & 25 & 33 \\
\hline AC Nanda ${ }^{b}$ & A & $\mathrm{G}$ & 54 & 75 \\
\hline Sumai- $3^{\mathrm{b}}$ & G & $\mathrm{T}$ & 35 & 47 \\
\hline
\end{tabular}

a DAI is days after inoculation.

b AC Nanda is the susceptible cultivar that was used to generate ethyl methane sulfonate variants and Sumai-3 is the resistant check cultivar. coincided with the stage of anthesis most vulnerable for FHB infection and progression (Bai and Shaner 2004).

A study of multiple $F$. graminearum isolates suggested that the 3ADON chemotypes were more aggressive than the $15-\mathrm{ADON}$ chemotypes based on disease development and DON production (Puri and Zhong 2010). Further, screening for FHB resistance using spike culture adequately differentiated FHB-resistant Sumai-3 and FHB-susceptible AC Nanda. The proposed in vitro method was completed by 11 DAI compared with disease scoring in in vivo methods, in which disease screening occurs at approximately 3 weeks after inoculation (Shin et al. 2014). The method is independent of environmental inconsistency and growth parameters can be controlled to support the plant and spike, as well as fungal growth. Spikes can be point inoculated with same concentration of inoculum to confirm a similar pathogen load. Therefore, the proposed method can be considered a rapid and reliable screening method for FHB disease resistance.

SCDV provides new sources of FHB resistance. Some FHB-resistant sources such as Sumai-3 are known; however, their use in breeding programs is limited due to poor adaptability to the local environment, inferior agronomic traits and low grain quality (McMullen et al. 2012). The SCDV derived from AC Nanda (Canada western soft white spring wheat) with high FHB resistance may overcome these difficulties and can be incorporated into breeding programs. The SCDV population (134 wheat variants) differed in FHB resistance, ranging from 15 to $100 \%$. Compared with Sumai-3, higher FHB resistance occurred in some SCDV and may be utilized as new sources of FHB resistance. Although no abnormal agronomic characteristic was observed while growing FHB-resistant lines in the greenhouse, their performance needs to be examined in field disease nurseries at multiple sites and over multiple years.

UDP-glucosyltransferase SNPs associated with FHB resistance and reduced DON accumulation. To develop new FHB-resistant cultivars, an understanding of the molecular mechanisms underlying FHB resistance is needed. The SNP detected in TaUGT-2B and TaUGT-3B were associated with FHB resistance in the SCDV population. The DON accumulation at various stages after $F$. graminearum inoculation in FHB-resistant and susceptible wheat genotypes confirmed the association with TaUGT-2B and TaUGT-3B, as well as with FHB resistance. Although higher DON accumulation was observed at 2 DAI in FHB-resistant genotypes compared with susceptible genotypes, induction of $U G T$ expression appears to limit further DON accumulation. Therefore, a gradual decrease in DON concentration was observed after 2 DAI in FHB-resistant wheat genotypes, which was associated with reduced FHB disease severity (Type II resistance). Most of the studies to date have indicated induced $U G T$ expression when plants were exposed to DON externally ( $\mathrm{Li}$ et al. 2015). The results of our study support this as we observed differences in the in vivo DON accumulation

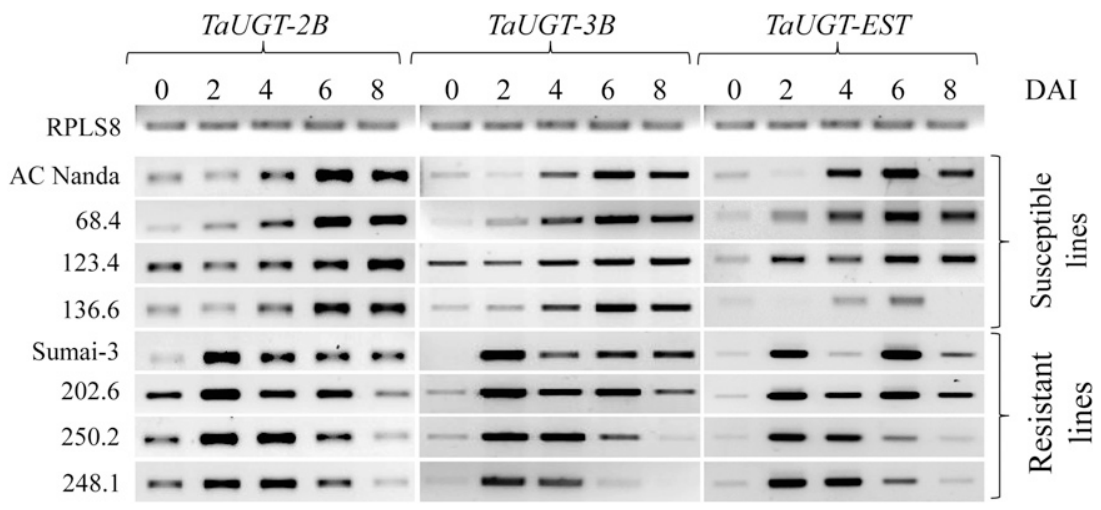

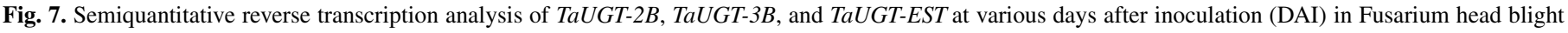
resistant and susceptible wheat genotypes. 


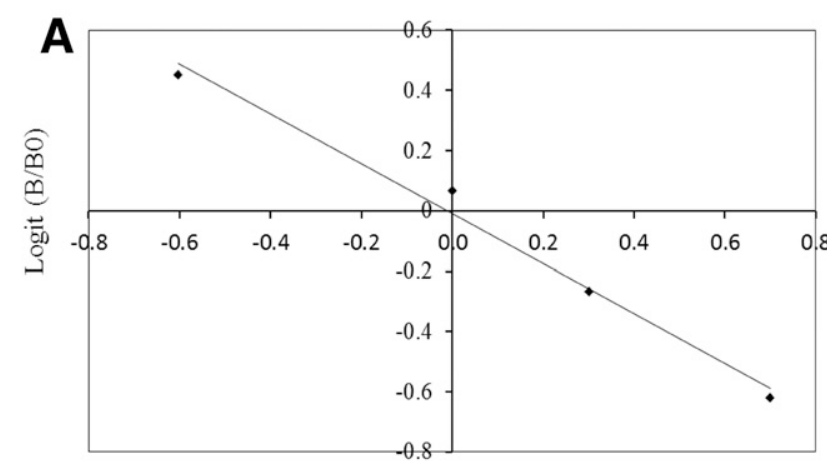

Log (Concentration)

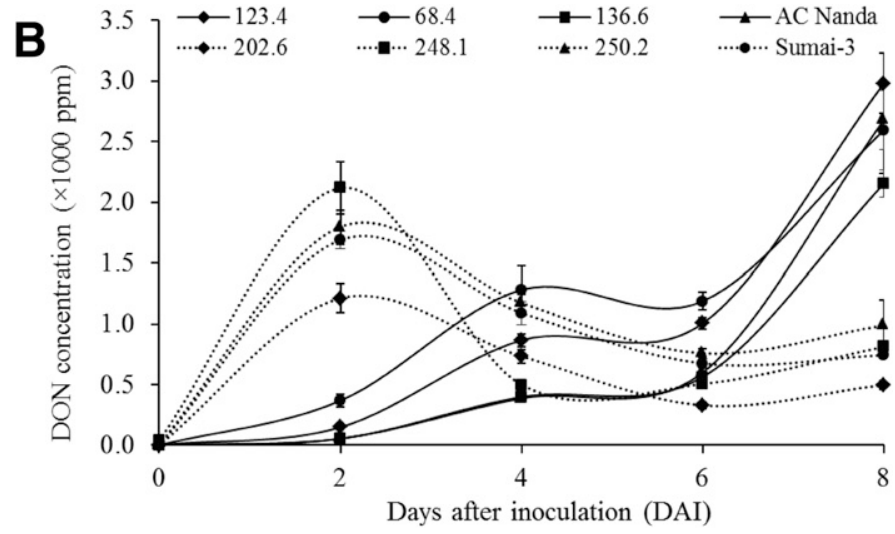

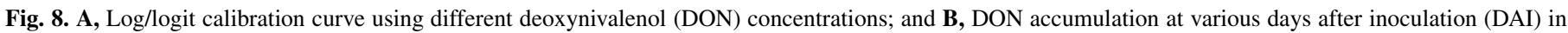
spikes of Fusarium head blight resistant and susceptible wheat genotypes.

pattern between FHB-resistant and susceptible wheat genotypes. $U G T$ transcript abundance was similar in all wheat genotypes immediately after inoculation (0 DAI), irrespective of FHB disease resistance. The DON may act as an inducer for TaUGT-2B and $T a U G T-3 B$ in wheat; hence in FHB-resistant genotypes, higher DON accumulation was observed that induced $U G T$ during the initial stages of disease development. In FHB-susceptible genotypes, low concentration of DON could not induce $U G T$ expression during the initial stages of disease induction. However, as the disease progressed both DON concentration and FHB expression increased gradually (Figs. 7 and 8). Maximum UGT expression at 6 or 8 DAI, in FHB-susceptible genotypes, could not restrict DON accumulation, which resulted in higher FHB disease severity. Expression of TaUGT-2B and TaUGT-3B clearly differentiated FHB-resistant from FHB-susceptible wheat genotypes. TaUGT-EST expression was also associated with FHB disease resistance except in SCDV 123.4. The induced expression of $U G T$ and their association with FHB resistance and DON accumulation concurs with previous studies (Li et al. 2015; Lin et al. 2008; Lulin et al. 2010). The present study also identified the participation of B-genome specific TaUGT-2 and Ta-UGT-3 in FHB resistance. Based on previous studies, Fhbl confers Type II FHB resistance, mainly by regulating cell wall thickening (Gunnaiah et al. 2012) or DON detoxification (Kluger et al. 2015; Lemmens et al. 2005). Combined genetic and biochemical evidence suggest that Fhbl includes a gene-for-UDP-glucosyl transferase (UGT) that conjugates DON to D3G or a gene that regulates UGT (Lemmens et al. 2005). A recent study reported a pore-forming, toxin-like (PFT) gene at the Fhbl locus that conferred enhanced FHB resistance in wheat (Rawat et al. 2016), and concluded that DON detoxification is independent of the PFT gene. The UDP-glycosyltransferase (UGT) genes were suggested to be present in the vicinity of the Fhbl locus contributing to FHB resistance by rapid DON detoxification to DON glycoside (Rawat et al. 2016). Consequently, several UDPglycosyltransferase (UGT) genes induced by FHB inoculation or DON treatment were differentially expressed in cultivars varying in FHB resistance (Li et al. 2015; Lin et al. 2008; Lulin et al. 2010). In $F$. graminearum-infected young spikes of bread wheat (Triticum aestivum L.), expression of TaUGT2 was induced, whereas expression of TaUGT1 was repressed suggesting that TaUGT2 may participate in wheat resistance to FHB (Lin et al. 2008). A DON-resistance related gene TaUGT3 was also cloned and characterized from a FHB-resistant wheat (cultivar Wangshuibai), overexpression of which improved DON tolerance in Arabidopsis (Lulin et al. 2010). Transgenic Arabidopsis (Shin et al. 2012) and wheat (Li et al. 2015) expressing a barley $U G T$, provided a high level of resistance to FHB by detoxifying DON to D3G. It is important to note that DON is not the only virulence factor of F. graminearum (Paranidharan et al. 2008); therefore, it is important to characterize the chemotype of the isolate.
The SNP found in the present study are synonymous; however, they can induce a significant phenotypic effect due to their impact on gene function (Chagné et al. 2008; Kimchi-Sarfaty et al. 2007; Palatnik et al. 2003). These 'silent' substitutions may regulate translation speed, protein homeostasis, metabolic fate, and sometimes posttranslational modifications (Shabalina et al. 2013).

In conclusion, the present study established the spike culture as a screening tool for FHB resistance. The spike culture method clearly differentiated FHB resistance of AC Nanda, Sumai-3, and 134 SCDV genotypes. The SNPs in TaUGT-2B and TaUGT-3B were associated with FHB resistance in wheat. The FHB-resistant wheat variants identified can be utilized as new genetic resources in wheat breeding programs. The SNP found in TaUGT-2B and TaUGT-3B are currently being used for marker assisted selection for FHB resistance in wheat (unpublished data). The HRM assay was also established as a tool to identify SNP. FHB resistance is a quantitative trait; the FHB-resistant SCDV genotypes can be used to identify and characterize candidate genes in wheat to improve FHB resistance.

\section{ACKNOWLEDGMENTS}

We thank the Cereal Research Centre, Agriculture and Agri-Food Canada, Winnipeg for providing F. graminearum isolates. Plant Gene Resources of Canada (PGRC, Saskatoon, SK, Canada) is acknowledged for providing seeds of Triticum monococcum (CN 37598), Aegilops speltoides (CN 45194), and Aegilops tauschii (CN 30812). Financial support from the Canada Research Chairs program and the Agricultural Development Fund of the Saskatchewan Ministry of Agriculture is gratefully acknowledged. C. Huang is a doctoral fellowship recipient of the China Scholarship Council.

\section{LITERATURE CITED}

Altschul, S. F., Gish, W., Miller, W., Myers, E. W., and Lipman, D. J. 1990. Basic local alignment search tool. J. Mol. Biol. 215:403-410.

Arruda, M. P., Brown, P., Brown-Guedira, G., Krill, A. M., Thurber, C., Merrill, K. R., Foresman, B. J., and Kolb, F. L. 2016. Genome-wide association mapping of Fusarium head blight resistance in wheat using genotypingby-sequencing. Plant Genome 9:1-14.

Bai, G., and Shaner, G. 1994. Scab of wheat-Prospects for control. Plant Dis. 78:760-766.

Bai, G., and Shaner, G. 2004. Management and resistance in wheat and barley to Fusarium head blight. Annu. Rev. Phytopathol. 42:135-161.

Brar, G. S., Dokken-Bouchard, F., Peluola, C., Sliva, T., Stephens, D., Singh, G., Kutcher, H. R., and Fernandez, M. R. 2016. Fusarium head blight in common and durum wheat in Saskatchewan in 2015. Can. Plant Dis. Surv. 96:117-119.

Buerstmayr, H., Ban, T., and Anderson, J. A. 2009. QTL mapping and markerassisted selection for Fusarium head blight resistance in wheat: A review. Plant Breed. 128:1-26.

Chagné, D., Gasic, K., Crowhurst, R. N., Han, Y., Bassett, H. C., Bowatte, D. R., Lawrence, T. J., Rikkerink, E. H., Gardiner, S. E., and Korban, S. S. 2008. Development of a set of SNP markers present in expressed genes of the apple. Genomics 92:353-358. 
Charmley, L. L., and Trenholm, H. L. 2015. Mycotoxins in livestock feed. RG8 Regulatory Guidance: Contaminants in Feed. Canadian Food Inspection Agency, Canada. http://www.inspection.gc.ca/animals/feeds/regulatoryguidance/rg-8/eng/1347383943203/1347384015909?chap=1

Cho, S. H., Lee, J., Jung, K. H., Lee, Y. W., Park, J. C., and Paek, N. C. 2012. Genome-wide analysis of genes induced by Fusarium graminearum infection in resistant and susceptible wheat cultivars. J. Plant Biol. 55:64-72.

Ganeshan, S., and Chibbar, R. N. 2014. Functional genomics analysis of grain quality using an in vitro immature wheat spike culture system. Pages 275-284 in: Biotechnology and Plant Breeding Perspectives. R. K. Behl and E. Arseniuk, eds. Agrobios International, Jodhpur, India.

Ganeshan, S., and Chibbar, R. N. 2017. A simple novel expedited spike culture-derived variation creation strategy in wheat. Cereal Res. Commun. doi.org/10.1556/0806.45.2017.047

Ganeshan, S., Drinkwater, J., Repellin, A., and Chibbar, R. N. 2010. Selected carbohydrate metabolism genes show coincident expression peaks in grains of in vitro-cultured immature spikes of wheat (Triticum aestivum L.). J. Agric. Food Chem. 58:4193-4201.

Ganeshan, S., Leis, M., Drinkwater, J. M., Madsen, L. T., Jain, J. C., and Chibbar, R. N. 2012. In vitro-cultured wheat spikes provide a simplified alternative for studies of Cadmium uptake in developing grains. J. Sci. Food Agric. 92:1740-1747.

Gunnaiah, R., Kushalappa, A. C., Duggavathi, R., Fox, S., and Somers, D. J. 2012. Integrated metabolo-proteomic approach to decipher the mechanisms by which wheat QTL (Fhb1) contributes to resistance against Fusarium graminearum. PLoS One 7:e40695.

Henriquez, M. A., de Rocquigny, P., Derksen, H., Miranda, D., and Gruenke, O. 2016. Fusarium head blight of spring wheat in Manitoba in 2015. Can. Plant Dis. Surv. 96:131-133.

Ichinoe, M., Kurata, H., Sigiura, Y., and Ueno, Y. 1983. Chemotaxonomy of Gibberrella zeae with special reference to production of trichothecenes and zearalenone. Appl. Environ. Microbiol. 46:1364-1369.

Kimchi-Sarfaty, C., Oh, J. M., Kim, I. W., Sauna, Z. E., Calcagno, A. M., Ambudkar, S. V., and Gottesman, M. M. 2007. A "silent" polymorphism in the MDR1 gene changes substrate specificity. Science 315:525-528.

Kluger, B., Bueschl, C., Lemmens, M., Michlmayr, H., Malachova, A., Koutnik, A., Maloku, I., Berthiller, F., Adam, G., Krska, R., and Schuhmacher, R. 2015. Biotransformation of the mycotoxin deoxynivalenol in Fusarium resistant and susceptible near isogenic wheat lines. PLoS One 10:e0119656.

Lemmens, M., Scholz, U., Berthiller, F., Dall'Asta, C., Koutnik, A., Schuhmacher, R., Adam, G., Buerstmayr, H., Mesterhazy, A., Krska, R., and Ruckenbauer, P. 2005. The ability to detoxify the mycotoxin deoxynivalenol colocalizes with a major quantitative trait locus for Fusarium head blight resistance in wheat. Mol. Plant-Microbe Interact. 18:1318-1324.

Li, X., Shin, S., Heinen, S., Dill-Macky, R., Berthiller, F., Nersesian, N., Clemente, T., McCormick, S., and Muehlbauer, G. J. 2015. Transgenic wheat expressing a barley UDP-glucosyltransferase detoxifies deoxynivalenol and provides high levels of resistance to Fusarium graminearum. Mol. Plant-Microbe Interact. 28:1237-1246.

Lin, F. Y., Lu, Q. X., Xu, J. H., and Shi, J. R. 2008. Cloning and expression analysis of two salt and Fusarium graminearum stress associated UDPglucosyltransferases genes in wheat. Yi Chuan 30:1608-1614.

Liu, S., Zhang, X., Pumphrey, M. O., Stack, R. W., Gill, B. S., and Anderson, J. A. 2006. Complex microcolinearity among wheat, rice, and barley revealed by fine mapping of the genomic region harboring a major QTL for resistance to Fusarium head blight in wheat. Funct. Integr. Genomics 6: 83-89.

Lulin, M., Yi, S., Aizhong, C., Zengjun, Q., Liping, X., Peidu, C., Dajun, L., and Xiu, E. W. 2010. Molecular cloning and characterization of an up-regulated UDP-glucosyltransferase gene induced by DON from Triticum aestivum L. cv. Wangshuibai. Mol. Biol. Rep. 37:785-795.
Maresca, M., Mahfoud, R., Garmy, N., and Fantini, J. 2002. The mycotoxin deoxynivalenol affects nutrient absorption in human intestinal epithelial cells. J. Nutr. 132:2723-2731.

McMullen, M., Bergstrom, G., De Wolf, E., Dill-Macky, R., Hershman, D., Shaner, G., and Van Sanford, D. 2012. A unified effort to fight an enemy of wheat and barley: Fusarium head blight. Plant Dis. 96:1712-1728.

Mesterházy, A. 1995. Types and components of resistance to Fusarium head blight of wheat. Plant Breed. 114:377-386.

Niwa, S., Kubo, K., Lewis, J., Kikuchi, R., Alagu, M., and Ban, T. 2014. Variations for Fusarium head blight resistance associated with genomic diversity in different sources of the resistant wheat cultivar 'Sumai 3'. Breed. Sci. 64:90-96.

Palatnik, J. F., Allen, E., Wu, X. L., Schommer, C., Schwab, R., Carrington, J. C., and Weigel, D. 2003. Control of leaf morphogenesis by microRNAs. Nature 425:257-263.

Paranidharan, V., Abu-Nada, Y., Hamzehzarghani, H., Kushalappa, A. C., Mamer, O., Dion, Y., Rioux, S., Comeau, A., and Choiniere, L. 2008. Resistance-related metabolites in wheat against Fusarium graminearum and the virulence factor deoxynivalenol (DON). Botany 86:1168-1179.

Parry, D. W., Jenkinson, P., and Mcleod, L. 1995. Fusarium ear blight (Scab) in small-grain cereals-A Review. Plant Pathol. 44:207-238.

Pestka, J. J., Zhou, H. R., Moon, Y., and Chung, Y. J. 2004. Cellular and molecular mechanisms for immune modulation by deoxynivalenol and other trichothecenes: Unraveling a paradox. Toxicol. Lett. 153:61-73.

Puri, K. D., and Zhong, S. 2010. The 3ADON population of Fusarium graminearum found in North Dakota is more aggressive and produces a higher level of DON than the prevalent 15ADON population in spring wheat. Phytopathology 100:1007-1014

Rawat, N., Pumphrey, M. O., Liu, S., Zhang, X., Tiwari, V. K., Ando, K., Trick, H. N., Bockus, W. W., Akhunov, E., Anderson, J. A., and Gill, B. S. 2016. Wheat $F h b 1$ encodes a chimeric lectin with agglutinin domains and a pore-forming toxin-like domain conferring resistance to Fusarium head blight. Nat. Genet. 48:1576-1580.

Sadasivaiah, R. S., Perkovic, S. M., Pearson, D. C., and Postman, B. 2000. Registration of AC Nanda wheat. Crop Sci. 40:579-580.

Shabalina, S. A., Spiridonov, N. A., and Kashina, A. 2013. Sounds of silence: Synonymous nucleotides as a key to biological regulation and complexity. Nucleic Acids Res. 41:2073-2094.

Sharma, P., Ganeshan, S., Fowler, D. B., and Chibbar, R. N. 2013. Characterization of two wheat enolase cDNA showing distinct patterns of expression in leaf and crown tissues of plants exposed to low temperature. Ann. Appl. Biol. 162:271-283.

Shin, S., Kim, K.-H., Kang, C.-S., Cho, K.-M., Park, C.-S., Okagaki, R., and Park, J.-C. 2014. A simple method for the assessment of Fusarium head blight resistance in Korean wheat seedlings inoculated with Fusarium graminearum. Plant Pathol. J. 30:25-32.

Shin, S., Torres-Acosta, J. A., Heinen, S. J., McCormick, S., Lemmens, M., Paris, M. P., Berthiller, F., Adam, G., and Muehlbauer, G. J. 2012. Transgenic Arabidopsis thaliana expressing a barley UDP-glucosyltransferase exhibit resistance to the mycotoxin deoxynivalenol. J. Exp. Bot. 63:4731-4740.

Snijders, C. H. A. 2004. Resistance in wheat to Fusarium infection and trichothecene formation. Toxicol. Lett. 153:37-46.

Talebi, A. B., Talebi, A. B., and Shahrokhifar, B. 2012. Ethyl methane sulphonate (EMS) induced mutagenesis in Malaysian rice (cv. MR219) for lethal dose determination. Am. J. Plant Sci. 3:1661-1665.

Waldron, B. L., Moreno-Sevilla, B., Anderson, J. A., Stack, R. W., and Frohberg, R. C. 1999. RFLP mapping of QTL for Fusarium head blight resistance in wheat. Crop Sci. 39:805-811.

Wetterhorn, K. M., Newmister, S. A., Caniza, R. K., Busman, M., McCormick, S. P., Berthiller, F., Adam, G., and Rayment, I. 2016. Crystal structure of Os79 (Os04g0206600) from Oryza sativa: A UDP-glucosyltransferase involved in the detoxification of deoxynivalenol. Biochemistry 55:6175-6186. 